\title{
GENERATION OF SUB-PICOSECOND ELECTRON BUNCHES FROM SUPERCONDUCTING 5.3 CELL RF GUN AND COHERENT WIGLER RADIATION
}

\author{
V.N.Volkov, BINP, Novosibirsk, Russia \\ D.Janssen, R.Wuensch, FZ-Rossendorf, Germany
}

\begin{abstract}
In this paper we discuss the possibility to generate subpicosecond electron bunches with a small emittance directly from the superconducting 5.3 cell $\mathrm{rf}$ gun [1] by illuminating a photo-cathode in the rf cavity with a phaselocked 3 ps laser pulses. The bunch length compression caused by the RF force take place in the first TESLA cell of the rf gun. Numerical simulations of the beam dynamics in the $\mathrm{rf}$ gun and of the radiation of the corresponding electron bunch in a wiggler predict a coherent enhancement of radiation for wavelength smaller than 100 micron.
\end{abstract}

\section{INTRODUCTION}

Sub-picosecond electron pulses have many scientific and industrial applications. One of them is the coherent enhancement of wiggler radiation. It requires injectors which can generate electron bunches with length of about 10 to $20 \mu \mathrm{m}$. A superconducting multi-cell photocathode rf gun may become the best variant of the such injector. Some main arguments in favour of this choice are the following: absence of high power RF generation, the strong coupling between cells makes this structure insensitive to mechanical errors and gives the structure the capability to operate at high acceleration gradient. The advantage for this scheme is its simplicity and compactness.

In the last years, our group works on the development of a superconducting RF gun injector [1]. Calculations of $1 / 2+3$ cells RF gun on basis of TESLA structure show good quality of a bunch [2] compatible for FEL requirements. The same structure with modified first cell can be used for getting of subpicosecond bunches. The length of first cell must be shorter than $1 / 2$ wavelength about 0.3 wavelength. The number of cells 5 is chosen to obtain bunch energy about $10 \mathrm{MeV}$. The sketch of the RF gun is shown on fig.1.

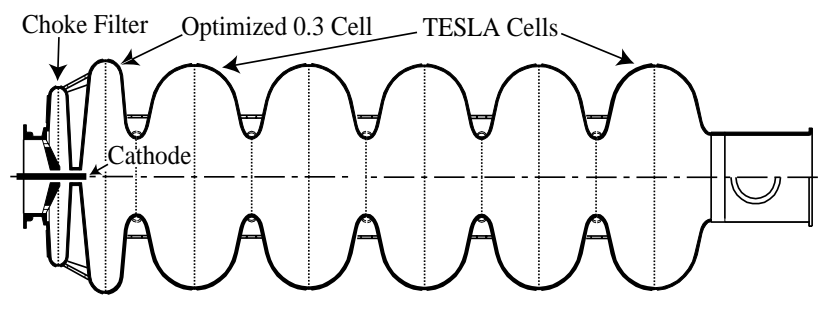

Figure1: Superconducting 5.3 cell RF gun injector.
Numerical simulation of this injector gives a rms bunch length 7.2 and $15.4 \mu \mathrm{m}$ correspondingly to 1 and $10 \mathrm{pC}$ bunch charge, when a laser pulse length is assumed to be 3 ps (FWHM).

\section{RF COMPRESSION INSIDE THE RF GUN CAVITY}

\subsection{Basic}

For well-known electron bunch compression method $[3,4]$, where the bunch launches at small RF phase and compresses in the first $1 / 2$ cell, we propose a new modification of this process. First thing, in this method the beam is launched not at small RF phase but at rather big values (about 30 degree in this calculation). Secondly, compression caused by the RF force occurs inside the full cell placed just after the first 0.3 cell of the RF gun cavity.

Due to a shorter length of 0.3 cell the bunch enters to the first full cell earlier, when there is a negative RF phase in the full cell. The bunch at the beginning of the first full cell partially decelerates and then accelerates again. The tail of the beam gains energy faster than the head. Electrons entered to the full cell at a later time receive higher acceleration than those entered earlier. The higher energy electrons then catch up with those at the head and reduce the bunch length.

There exists an optimized entering phase at which the bunch length is at a minimum. The dependence of this phase (named optimal phase) on initial bunch energy at the beginning of the full cell is shown in the fig.2a. If the entering phase will be more than the optimal phase then the head and tail of the bunch switch the roles

\subsection{Single-particle beam dynamics}

In this section we study the electron-beam dynamics in a part of rf gun structure consisting of full cells only. The first cell may has a various form with a suitable length and RF field strength to get a needed energy and rf phase of a bunch at its exit. The rf field in a cavity accelerates electrons entered to the full cells with some initial energy. The electric field along the axis will be assumed to be of the following simple form:

$E_{z}=E_{0} \cdot \sin (k z) \cdot \sin \left(\omega t+\varphi_{o}\right)$

Here $E_{o}$ is the peak-accelerating field. $\lambda$ is the $\mathrm{rf}$ wavelength, $k=2 \pi / \lambda$ and $\varphi_{0}$ is the rf phase as the particle enter the structure at $\mathrm{z}=0, \mathrm{t}=0$. 


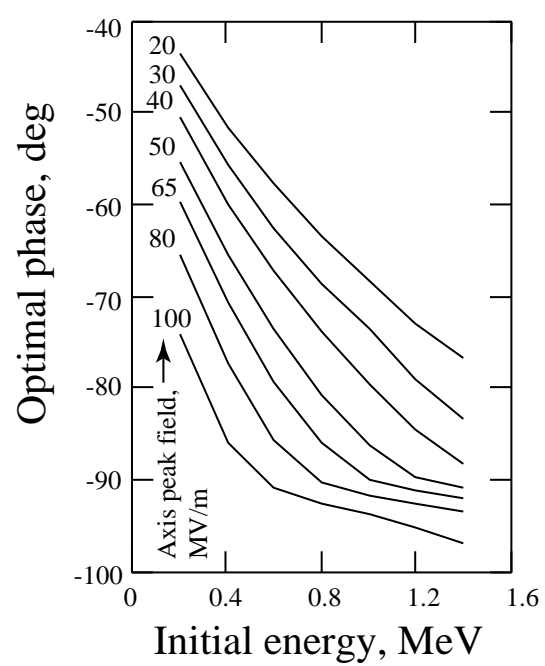

a)

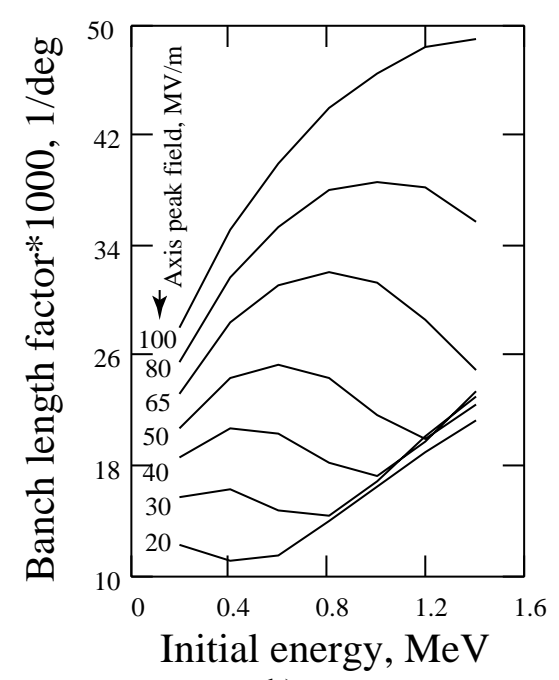

b)

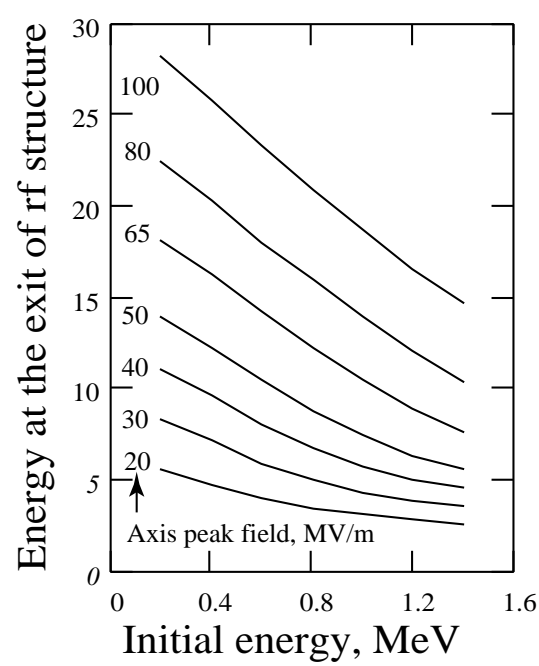

c)

Figure 2: Electron-beam dynamics in 5 full cell RF structure with simple form of electric field along the axis (1). The initial bunch energy assumed to be at the entrance of rf structure.

a) Optimized entering phase at which the bunch length at the exit of rf structure is at a minimum; b) Bunch length factor from equation (2); c) Bunch energy at the exit of rf structure. Here RF frequency is assumed to be $1300 \mathrm{MHz}$, so if Eo = $100 \mathrm{MV} / \mathrm{m}$ we have $\alpha=\mathrm{eE}_{\mathrm{o}} / 2 \mathrm{mc}^{2} \mathrm{k}=3.591$. Each curve in the pictures remains the same for another rf frequency if a corresponding $\alpha$ is constant [4].

Solving the relativistic equation describing the longitudinal motion of electron in this rf field (see fig.2a) we find that there always exist an optimal phase at which the bunch length is a minimum for wide range of initial bunch energy. If bunch charge is negligible and initial bunch length $\left(\sigma_{\text {init }}\right)$ small enough the minimum bunch length at the rf gun exit $\left(\sigma_{\text {exit }}\right)$ can be expressed as:

$\sigma_{\text {exit }}=\Lambda \cdot \sigma_{\text {init }}^{2}$

The factor $\Lambda$ (named as bunch length factor) is shown in fig. $2 b$, i.e. $\Lambda$ present a bunch length at the RF gun exit in degree when $\sigma_{\text {init }}=1^{\circ}$ of $\mathrm{rf}$ phase.

The bunch energy at the exit of RF gun depending on initial energy of the bunch at the entrance of the cavity is shown in fig.2c.

\section{NUMERICAL SIMULATION}

In the simulation presented here, a laser pulse duration (FWHM) of 3 ps with a Gaussian distribution is assumed as well as an instantaneous response of the cathode. The total electron charge in a bunch is assumed to be 1 and 10 pC. The RF field distribution in the RF gun axis is shown in fig.3. The beam dynamics is simulated using PARMELA code. The result is shown in table1.

\subsection{Sensitivity to a parameter changing}

The injection phase into the RF gun as well as the cathode radius is carefully adjusted to get a minimum of bunch length. The sensitivity of compressed bunch length $(\delta \mathrm{L})$ to the change of the injection phase $(\delta \varphi)$ and cathode radius $\left(\delta R_{c}\right)$ may be expressed as:

$\delta \mathrm{L}[\mu \mathrm{m}]=2.8 \cdot \delta \varphi^{2}[\mathrm{deg}] \quad$ and $\delta \mathrm{L}[\mu \mathrm{m}]=0.014 \cdot \delta \mathrm{R}_{\mathrm{c}}^{2}[\mu \mathrm{m}]$.

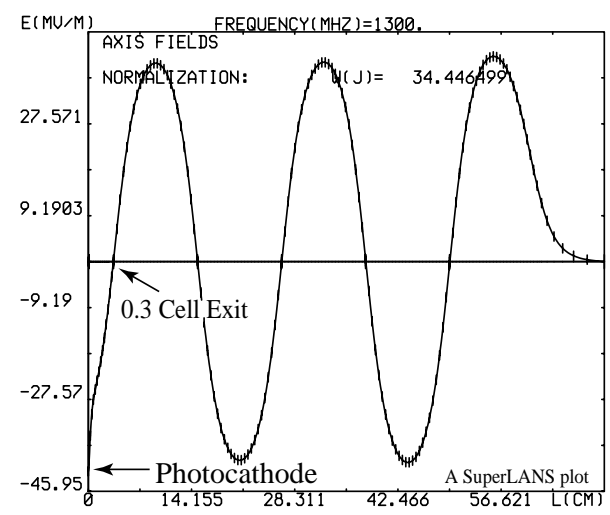

Fig.3. RF field distribution in the 5.3 cell RF gun axis

Table1. Bunch characteristics of 5.3 cell rf gun (at a distance of $0.5 \mathrm{~m}$ from $\mathrm{rf}$ gun exit).

\begin{tabular}{|l|c|c|}
\hline Bunch charge, $\mathrm{pC}$ & 1 & 10 \\
\hline Laser spot size, mm & 2.0 & 2.72 \\
\hline Injection RF phase, deg & 30.7 & 27.8 \\
\hline Bunch energy, MeV & 9.74 & 9.74 \\
\hline Energy spread (rms), keV & 7.25 & 6.76 \\
\hline $\begin{array}{l}\text { Transversal normalized } \\
\text { emittance, mm·mrad }\end{array}$ & 0.42 & 1.25 \\
\hline $\begin{array}{l}\text { Longitudinal emittance, } \\
\text { mm.keV }\end{array}$ & 0.047 & 0.102 \\
\hline Bunch length $(\mathbf{r m s}), \boldsymbol{\mu m}$ & $\mathbf{7 . 2}$ & $\mathbf{1 5 . 4}$ \\
\hline
\end{tabular}




\subsection{Radiation}

An electron bunch passing through a planar undulator spontaneously emits electromagnetic coherent radiation [6]. Table 2 shows the calculated radiation power and radiation wavelength, which can be obtained with the ELBE undulators U27 and U50. The undulator characteristics are presented there.

Table 2. Characteristics of electromagnetic coherent radiation obtained from U27 and U50 undulators with average electron beam current of $1 \mathrm{~mA}$.

\begin{tabular}{|l|c|c|c|c|}
\hline Undulator name & \multicolumn{2}{|c|}{ U27 } & \multicolumn{2}{c|}{ U50 } \\
\hline Undulator period, mm & \multicolumn{2}{|c|}{27} & \multicolumn{2}{c|}{50} \\
\hline Undulator parameter $\mathrm{K}_{\mathrm{rms}}$ & \multicolumn{2}{|c|}{0.95} & \multicolumn{2}{c|}{1.2} \\
\hline Bunch charge, $\mathrm{pC}$ & \multicolumn{1}{|c|}{1} & 10 & 1 & 10 \\
\hline $\begin{array}{l}\text { Radiation wavelength, } \\
\mu \mathrm{6m}\end{array}$ & $\mathbf{6 4}$ & $\mathbf{6 4}$ & $\mathbf{1 5 2}$ & $\mathbf{1 5 2}$ \\
\hline Radiation power, W & $\mathbf{2 . 9 7}$ & $\mathbf{4 . 8 4}$ & $\mathbf{3 . 5 6}$ & $\mathbf{2 5 . 3}$ \\
\hline
\end{tabular}

\section{ACKNOWLEDGMENTS}

We wish to thank A.Pilan for assistance in translation into English.

\section{REFERENCES}

[1] D.Janssen etc., On the way to a superconducting $R F$ gun: first measurements with the gun cavity, Nucl. Instr. And Meth. In Phis. Res. A445 (2000) 408-412.

[2] D.Janssen, V.Volkov, RF focussing - an instrument for beam quality improvement in superconducting $R F$ guns, Nucl. Instr. And Meth. In Phis. Res. A452 (2000) 34-43.

[3] X.J.Wang, I.Ben-Zvi, Longitudinal emittance compensation in a photocathode rf gun injector, PAC97, 1997, 2793-2795.

[4] L.Serafini, R.Zhang, C.Pelegrini, Generation of subpicosecond electron bunches from $R F$ photoinjectors, Nucl. Instr. And Meth. In Phis. Res. A387 (1997) 305314.

[5] K.J.Kim, RF and Space-Charge Effects in Laserdriven RF Electron Guns . Nucl. Instr. And Meth. In Phis. Res. A275 (1989) 206.

[6] H.Wiedemann, Particle Accelerator Physics, SpringerVerlag Berlin, Heidelberg 1993 